\title{
Properties of Electrostatically-Driven Granular Medium: Phase Transitions and Charge Transfer
}

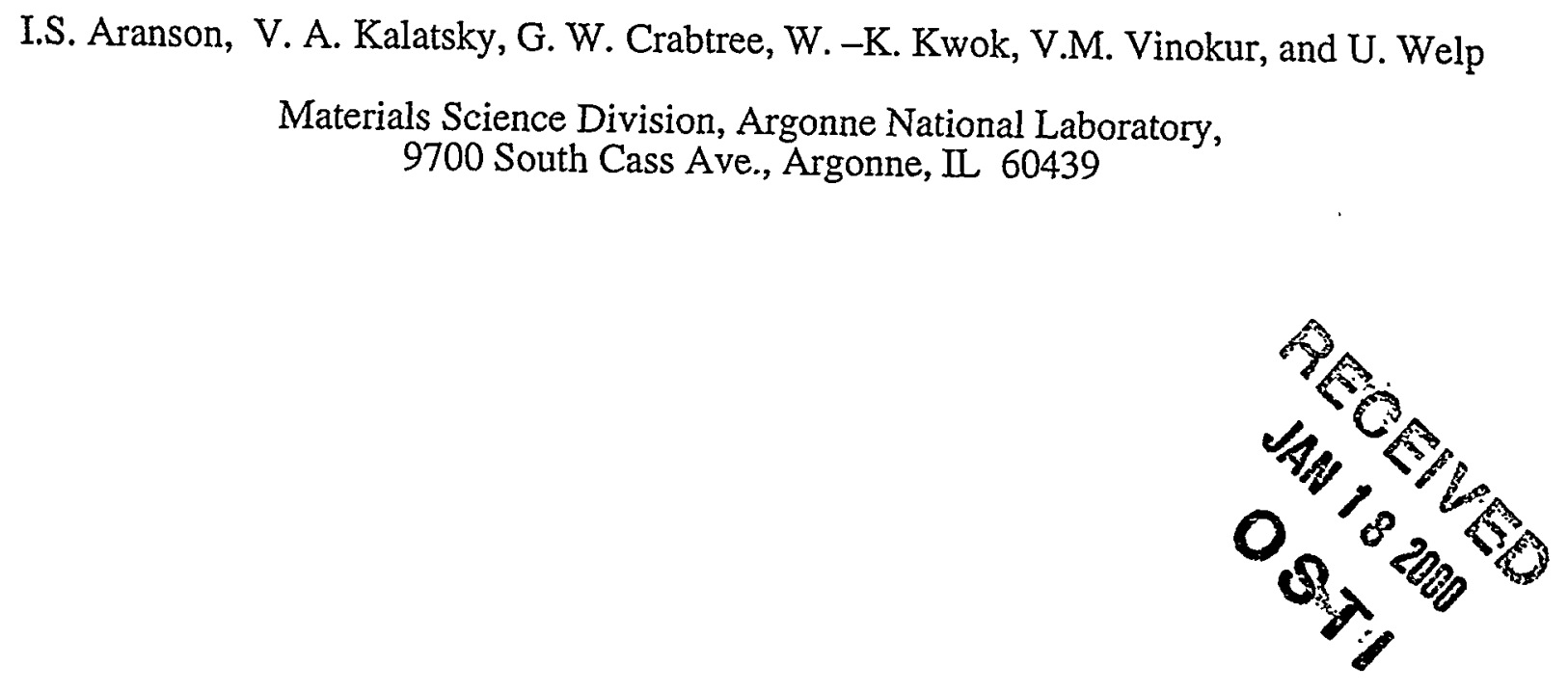

Proceedings of STAIF-2000, Albuquerque New Mexico

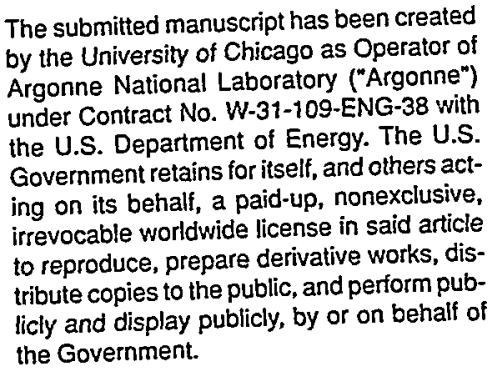

This work was supported by the U.S. Department of Energy, Under Contract No. W-31-109ENG-38 and by NSF, STCS \#DMR91-20000. 


\section{DISCLAIMER}

This report was prepared as an account of work sponsored by an agency of the United States Government. Neither the United States Government nor any agency thereof, nor any of their employees, make any warranty, express or implied, or assumes any legal liability or responsibility for the accuracy, completeness, or usefulness of any information, apparatus, product, or process disclosed, or represents that its use would not infringe privately owned rights. Reference herein to any specific commercial product, process, or service by trade name, trademark, manufacturer, or otherwise does not necessarily constitute or imply its endorsement, recommendation, or favoring by the United States Government or any agency thereof. The views and opinions of authors expressed herein do not necessarily state or reflect those of the United States Government or any agency thereof. 


\section{DISCLAIMER}

Portions of this document may be illegible in electronic image products. Images are produced from the best available original document. 


\title{
Properties of Electrostatically-Driven Granular Medium: Phase Transitions and Charge Transfer
}

\author{
I.S. Aranson, D. Blair, V. A. Kalatsky, G.W. Crabtree, W.-K. Kwok, \\ V.M. Vinokur and U. Welp \\ Materials Science Division, Argonne National Laboratory, \\ 9700 South Cass Avenue, Argonne, IL 60439
}

phone: (630)-252-9725; e-mail: aronson@vortex.msd.anl.gov

\begin{abstract}
The experimental and theoretical study of electrostatically driven granular material are reported. It is shown that the charged granular medium undergoes a hysteretic first order phase transition from the immobile condensed state (granular solid) to a fluidized dilated state (granular gas) with a changing applied electric field. In addition a spontaneous precipitation of dense clusters from the gas phase and subsequent coarsening - coagulation of these clusters is observed. Molecular dynamics simulations shows qualitative agreement with experimental results.
\end{abstract}

\section{INTRODUCTION}

The dynamics of granular materials with their strong contact interactions and inelastic collisions between grains poses a challenge for physicists and engineers. ${ }^{1-5}$ Peculiar properties due to new fascinating collective behaviors appear when small particles acquire an electric charge and respond to competing long-range electromagnetic and short range contact forces. Although of prime practical importance the properties of charged granular media and their response to the external field are still not fully understood.

The electrostatic excitation of granular medium offers unique new opportunities compared to traditional vibration techniques which have been developed to explore granular dynamics. ${ }^{3-7}$ It enables one to deal with extremely fine powders under low gas pressure or zero gravity conditions. Fine particles are more sensitive to electrostatic forces which arise through particle friction or space charges. ${ }^{8}$ Their large surface to volume ratio amplifies the effect of water or other surfactants. These effects intervene in the dynamics causing agglomeration, charging, cohesion etc, making mechanical experiments uncontrollable. Electrostatic driving utilizes these bulk forces, and allows also for control of dynamic behaviors by long-range electric forces. Investigation of the collective dynamics of very small particles has an additional advantage of being attained to "thermodynamically large" ensembles of about $10^{7}$ grains.

With this paper, we present our experimental and theoretical study of electrostatically driven granular material. ${ }^{9}$ We show that the charged granular medium undergoes a hysteretic first order phase transition from the immobile condensed state (granular solid) to a fluidized dilated state (granular gas) with a changing applied electric field. Charging of the particles results in electric current and possible increase of heat transfer. This current vanishes at the onset of particle motion at the critical field and grows gradually with the increase of the filed. In addition we observe a spontaneous precipitation of dense clusters from the gas phase. These clusters then evolve via coarsening dynamics: small clusters disappear and large clusters grow. Eventually the large isolated clusters assume almost perfect circular form. In the process of coarsening the electric current also changes in time due to decrease of particles in the gas phase. We establish that the origin of coarsening dynamics and hysteresis is due to a screening of the electric field in dense particle clusters. Our results show surprisingly high sensitivity of the phase boundaries to surface coating of the grains due to humidity.

\section{EXPERIMENTAL SET-UP}

The experimental cell is shown in Figure 1. Conducting particles are placed between the plates of a large capacitor which is energized by a constant or alternating electric field. To provide optical access to the cell, the upper conducting plate is made transparent. We used $4 \times 6 \mathrm{~cm}$ capacitor plates with a spacing of 1.5 
$\mathrm{mm}$ and $35 \mu \mathrm{m}$ copper powder. The field amplitude varied from 0 to $10 \mathrm{kV} / \mathrm{cm}$ and the frequencies on the interval of 0 to $250 \mathrm{~Hz}$. The representative number of particles in the cell was about $10^{7}$. The experiments were performed both in atmospheric pressure and in a high vacuum $\left(5 \times 10^{-5}\right.$ Torr $)$.

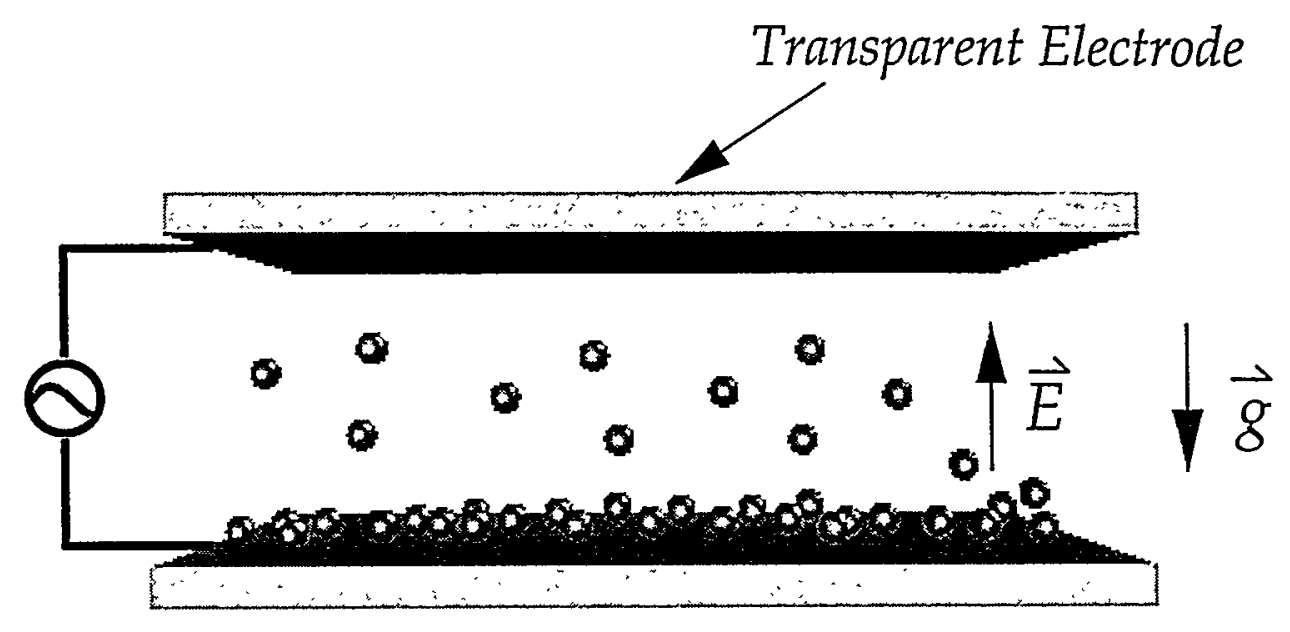

FIG. 1. The electrostatic cell

Let us first discuss the mechanism bringing particles into the motion. When the conducting particles come in contact with the capacitor plate they acquire a surface electric charge. As the magnitude of the electric field in the capacitor exceeds the critical value $E_{1}$ the resulting (upward) electric force overcomes the gravitational force $m g$ ( $m$ is the mass of the particle, $g$ is the acceleration due to gravity) and pushes the charged particles upward. When the grains hit the upper plate, they deposit their charge and fall back. Applying an alternating electric field $E=E_{0} \sin (2 \pi f t)$, and adjusting its frequency $f$ one can control the particle elevation by effectively turning them back before they collide with the upper plate.

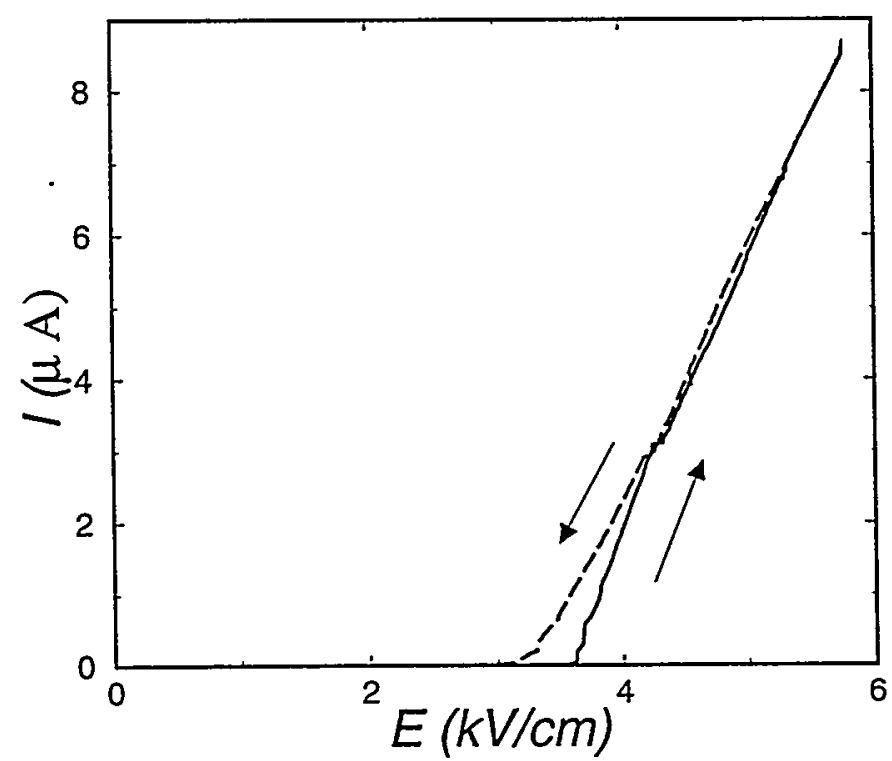

FIG. 2. The electric current $I$ across the cell vs applied electric field $E$. Solid line is obtained by increasing $E$ from zero to $E_{\max }$, dashed line is obtained by decreasing the field from $E_{\max }$ to zero. 
Charging and discharging of the particles results in electric current across the cell. This current vanishes at the onset of particle motion at the first critical field $E_{1}$ and grows gradually with the increase of $E$, as it can be seen from Figure 2. For the value of $E$ about $2 E_{1}$ it achieves $2-3 \mu$ a. The phase diagram for the experimental cell is shown in Figure 3. Isolated particles start to move at $E>E_{1}$. We have further found that at amplitudes of the electric field above a second threshold value, $E_{2}>E_{1}$, the granular medium forms a uniform gas-like phase (granular gas). This second field $E_{2}$ is $50-70 \%$ larger then $E_{1}$ in nearly the whole range of the parameters used. This hysteresis can be seen from the charge transport measurements shown in Figure 2: the electric current corresponding increasing of the electric field from zero to $E_{m a x}$ is smaller then the current for the decreasing field.

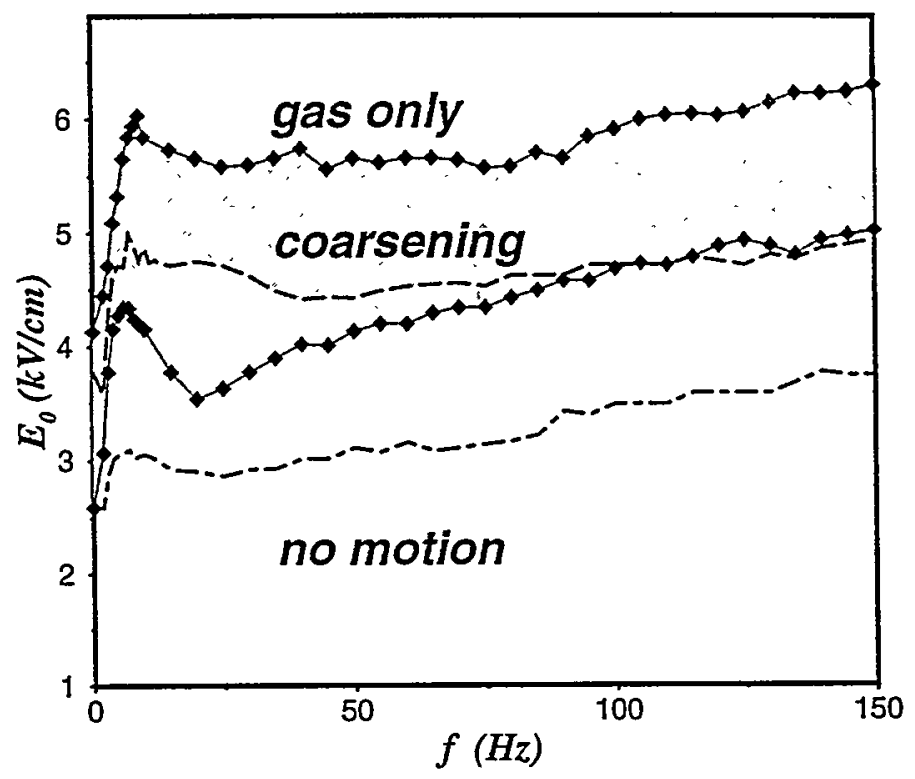

FIG. 3. The phase diagram as a function of amplitude and frequency of the alternative field $E=E_{0} \sin (2 \pi f t)$. Solid lines with diamonds show $E_{1,2}$ in open cell. Dashed line shows $E_{2}$ and dash-dotted line shows $E_{1}$ for the evacuated cell correspondingly. In the gas only domain clusters do not form spontaneously, in the coarsening domain both clusters and gas coexist.

In the field interval $E_{1}<E<E_{2}$, a remarkable phenomenon analogous to coalescence dynamics in systems exhibiting first order phase transitions is observed. ${ }^{10-12}$ Upon decreasing the field below $E_{2}$, the gas phase looses its stability and small clusters of stationary particles, containing about $10^{2}$ grains, surrounded by the granular gas spontaneously form. These clusters then evolve via coarsening dynamics: small clusters disappear and large clusters grow. Eventually the large isolated clusters assume almost perfect circular form (Figs 4a-c). In the process of coarsening the electric current also changes in time due to decrease of particles in the gas phase. After a very large time $(t \approx 30000 \mathrm{sec})$ only one large cluster containing about $10^{6}$ grains survives. Even at the final stage, the coexistence of phases is preserved - not all the particles join the last cluster. On the contrary, a dynamic equilibrium between the granular solid in the cluster and the surrounding gas takes place.

For the cell under atmospheric pressure (open cell) we find that both fields $E_{1}$ and $E_{2}$ grow as the function of frequency for large $f$ and show non-monotonous behavior for $f \approx 12 \mathrm{~Hz}$. This indicates a characteristic time of the order $100 \mathrm{msec}$. We suggest that cohesion may be responsible for this relatively large time. Indeed, due to the humidity of the air a surface coating should exist, thus giving meaning to some characteristic time $\tau$ for the grain to detach from the capacitor plate. Although the cohesive forces are very small, they become comparable with the gravitational/electric forces. In order to reduce the cohesion we evacuated the cell to very low pressure. As demonstrated from Fig. 3, the frequency dependence is indeed substantially reduced and becomes almost fiat. Small oscillations in the dependence for low frequency are probably due to a residual coating on the particles which does not completely evaporate in vacuum. 

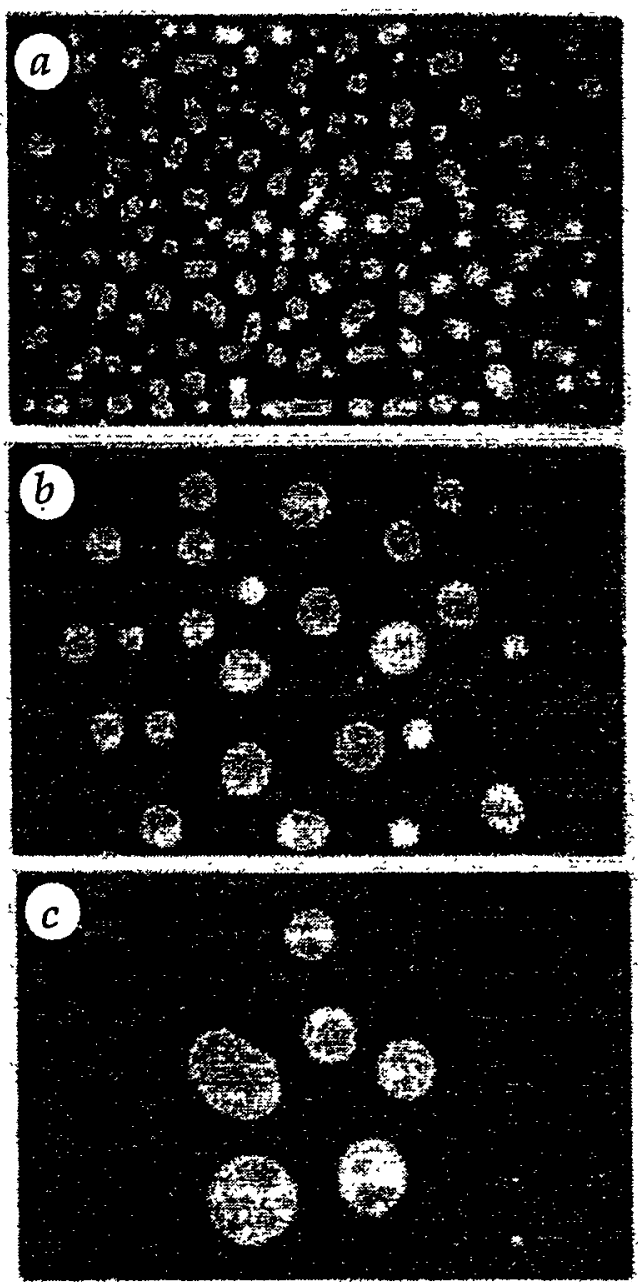

FIG. 4. Top view of the experimental cell for the moments of time (a) $t=1 \mathrm{sec}$, (b) $t=10 \mathrm{sec}$, and (c) $t=10910$ sec for $f=40 \mathrm{~Hz}$ and $E=5.25 \mathrm{kV} / \mathrm{cm}$.

\section{THEORETICAL MODEL}

To clarify the mechanism of coarsening and the observed hysteresis we compare the forces exerted on an individual particle and on a particle which is held within a cluster at the same applied electric field.
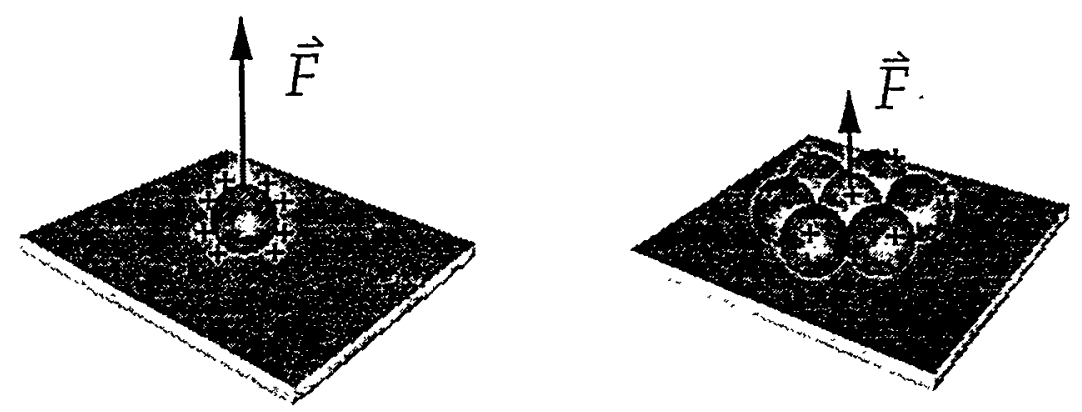

FIG. 5. The forces acting on individual particle and the particle in the cluster. Small pluses show the surface charges. 
The force between an isolated sphere and the capacitor plate in contact can be found as a limit of the problem of two conducting spheres when the radius of one sphere goes to infinity. ${ }^{13}$ Building on the technique of Ref. ${ }^{13}$, we arrive at the force $F_{e}$ in question:

$$
F_{e}=1.36 a^{2} E^{2}
$$

where $a$ is the radius of the sphere and $E$ is the field in the capacitor. The constant 1.36 comes from summing of infinite series. ${ }^{13}$ The electric force $F_{e}$ has to counterbalance the gravitational force $G=4 / 3 \pi \rho g a^{3}$, where $\rho$ is the density of the material. Comparing $F_{e}$ and $G$ we find the first critical field $E_{1}$ :

$$
E_{1}=\sqrt{\frac{4 \pi \rho g a}{3 \cdot 1.36}}
$$

Our theory indicates no frequency dependence of $E_{1}$. For the parameters of our experiments we obtain $E_{1}=2.05 \mathrm{kV} / \mathrm{cm}$, which is below than the experimental critical field $E_{1} \approx 2.4 \mathrm{kV} / \mathrm{cm}$. This discrepancy seems reasonable since we did not take into account additional molecular and/or contact forces which will increase the critical field.

If the spheres are in contact with each other, the surface charge will redistribute: each sphere in the cluster acquires smaller charge than that of an individual sphere due to a screening of the field by its neighbors. The exact derivation of the force acting on the particle within the cluster is not available at present. The upper bound can be obtained by replacing the square lattice of spheres with radius $a$ by a lattice of squares with area $4 a^{2}$. Since the charge density of flat layer is $\sigma=E / 4 \pi$ and the total electric force on the square is $F_{2}=4 a^{2} \sigma E / 2$. Thus we obtain the ratio of the fields to lift individual particle $E_{1}$ and the particle in the square lattice $E_{2}$ (note again no frequency dependence):

$$
E_{2} / E_{1}=\sqrt{2 \pi \cdot 1.36}=2.92 .
$$

For closed-packed hexagonal lattice one obtains a slightly higher value $E_{2} / E_{1} \approx 3.14$. The ratio of critical fields is independent of particle size and the density. For the constant applied field $E$, and, correspondingly, for the low frequency ac field, the ratio of the fields is about 3 . This is consistent with the experimental findings, see Fig. 3, although exceeds experimental value by the factor of 2 . More accurate account for the surface shape will reduce the ratio of the critical fields.

In order to study the details of coarsening process we performed molecular dynamics simulations. Several simplifications had been implemented: we neglected polarization effects of the particles and assumed that the charge is always located in the center of conducting sphere; we considered collisions between the particles and particle and conducting plate completely inelastic. The simulations show qualitative agreement with experiment: coarsening and first order phase transition between granular gas and granular solid. ${ }^{9}$

\section{ACKNOWLEDGEMENTS}

We thank Leo Kadanoff, Thomas Witten, Lorenz Kramer and Baruch Meerson for useful discussions. This research is supported by US DOE, grant W-31-109-ENG-38, and by NSF, STCS \#DMR91-20000. 


\section{REFERENCES}

' Jaeger, H.M., Nagel, S.R., and Behringer, R.P., "The Physics of Granular Materials," Physics Today 49, 32-38 (1996); "Granular Solids, Liquids, and Gases," Rev. Mod. Phys. 68, 1259-1273 (1996).

2 Kadanoff, L., "Built Upon Sand: Theoretical Ideas Inspired by Granular Flows," Rev. Mod. Phys. 71, 435-444 (1999)

${ }^{3}$ Melo F., Umbanhowar, P.B., and Swinney, H.L., "Hexagons, Kinks, and Disorder in Oscillated Granular Layers," Phys. Rev. Lett. 75, 3838-3841 (1995); Umbanhowar, P.B., Melo F., and Swinney, H.L., "Localized Excitations in a Vertically Vibrated Granular Layer," Nature 382, 793-796 (1996); "Periodic, Aperiodic, and Transient Patterns in Vibrated Granular Layers", Physica A 249, 1-9 (1998).

${ }^{4}$ Hunt, M.L., Weathers, R.C., Lee, A.T., Brennen, C.E., Wassgren C.E, "Effects of horizontal vibration on hopper flows of granular materials," Physics of Fluids 11, 68-75 (1999)

${ }^{5}$ Aranson, I.S., Blair, D., Kwok, W.-K., Karapetrov, G., Welp, U., Crabtree, G.W., Vinokur, V.MI., and Tsimring, L.S., "Controlled Dynamics of Interfaces in a Vibrated Granular Layer," Phys. Rev. Lett. 82, 731-734 (1999).

${ }^{6}$ Boothroyd, R.G. Flowing Gas-Solid Suspention, Chapman \& Hall, 1971;. Iinoya, K., Gotoh, K., and Higashitani, K., Powder Technology Handbook, N. Y. Marcel Dekker, 1990; MVitsuya, T. and Hunt, M.L., "Toner Particle Packing in an Electrostatic Field and its Effect on Heating in Electrophotography," Powder Technology 92, 119-125 (1997).

${ }^{7}$ Tennakoon, S.G.K., and Behringer, R.P, "Vertical and Horizontal Vibration of Granular Materials: Coulomb Friction and a Novel Switching State," Phys. Rev. Lett. 81, 794-797 (1998)

${ }^{8}$ M.E. Fayed and L. Otten, ed., Handbook of Powder Science \& Technology, second ed., Chapman \& Hall, 1997.

${ }^{9}$ Aranson, I.S., Blair, D., Kalatsky, V.A., Crabtree, G.W., Kwok, W.-K., Vinokur V.M., and Welp, U., "Electrostatically-Driven Granular Media: Phase Transitions and Coarsening," submitted to Nature, 1999.

${ }^{10}$ Lifshtz, E.MI., and Pitaevsky, L.P., Physical Kinetics, Pergamon, London, 1981.

${ }^{11}$ Giron, B., Meerson, B. and Sasorov, P.M., "Weak Selection and Stability of Localized Distributions in Ostwald Ripening," Phys. Rev. E 58, 4213-4116 (1998).

${ }^{12}$ Aranson, I.S., Meerson, B., and Sasorov, P.M., " Front-Curvature Effects in the Dynamics of Confined Radiatively Bistable Plasmas: Perfect Patterns and Ostwald Ripening," Phys. Rev. E 52, 948-971 (1995).

${ }^{13}$ Thomson, W., "On the Mutial Attraction or Repulsion between Two Electrified Spherical Conductors," Phil. Mag. s. 4, 5, 287-297 (1853); Jeans, J. The Mathematical Theory of Electricity and Magnetism, Cambridge University Press, Vth Ed., 1948 